\title{
Baryon femtoscopy considering residual correlations as a tool to extract strong interaction potentials
}

\author{
Maciej Szymański ${ }^{1, a}$, Adam Kisiel ${ }^{1}$, and Hanna Zbroszczyk ${ }^{1}$ \\ ${ }^{1}$ Warsaw University of Technology, Faculty of Physics
}

\begin{abstract}
In this article, the analysis of baryon-antibaryon femtoscopic correlations is presented. In particular, it is shown that taking into account residual correlations is crucial for the description of $\mathrm{p} \bar{\Lambda}$ and $\overline{\mathrm{p}} \Lambda$ correlation functions measured by the STAR experiment in $\mathrm{Au}-\mathrm{Au}$ collisions at the centre-of-mass energy per nucleon pair $\sqrt{s_{\mathrm{NN}}}=200 \mathrm{GeV}$. This approach enables to obtain $\mathrm{p} \bar{\Lambda}(\overline{\mathrm{p}} \Lambda)$ source size consistent with the sizes extracted from correlations in $\mathrm{p} \Lambda(\overline{\mathrm{p}} \bar{\Lambda})$ and lighter pair systems as well as with model predictions. Moreover, with this analysis it is possible to derive the unknown parameters of the strong interaction potential for baryon-antibaryon pairs under several assumptions.
\end{abstract}

\section{Introduction}

These proceedings are based on [1] where all the relevant details may be found.

Femtoscopy, the study of particle-correlations at low relative momentum, is a powerful tool extensively used in heavy-ion, proton-nucleus and proton-proton collisions. Such correlations arise due to Quantum Statistics (in case of identical particles) as well as Coulomb and strong Final State Interactions (FSI). The applications of this method include analysis of space-time structure at freeze-out, sensitivity to dynamics, quantum coherence of particles and parameters of interaction potentials [2-4]. Correlations of pions, kaons and protons were measured in STAR and ALICE and showed decrease of the radius with transverse mass [5-9]. However, STAR measurement of $\mathrm{p} \bar{\Lambda}$ and $\overline{\mathrm{p}} \Lambda$ correlations demonstrated that radius for these systems is about twice smaller than the one for the $\mathrm{p} \Lambda$ system [10]. This is in disagreement with hydrodynamics model $[11,12]$ and measurements for lighter pairs. In [10] residual correlations are mentioned as a vital aspect of baryon femtoscopy, but they are not considered in the analysis.

In this work we show that detailed consideration of residual correlations might be the solution of the small $\mathrm{p} \bar{\Lambda}$ radius measured in the STAR experiment. In addition, an attempt is made to derive the parameters of the strong interaction potential following the innovative method used in $[10,13]$. Especially, imaginary part of the scattering length is of special interest as it parametrises baryon-antibaryon annihilation process at low relative momentum. It is worth emphasising that strong interaction parameters in baryon-antibaryon systems are unknown apart from $\bar{p} \bar{p}, \mathrm{p} \overline{\mathrm{n}}$ and $\overline{\mathrm{p}} \mathrm{d}$ [14]. Also, there is a very little input from theory. Therefore, putting any constraints on these parameters is essential, particularly in light of recent observations of the deviations of proton yields from ALICE data with respect to thermal models [15].

\footnotetext{
ae-mail: maszyman@if.pw.edu.pl
} 


\section{Femtoscopic methodology}

Two-particle femtoscopic correlation function is expressed by the following equation [16]:

$$
C\left(\boldsymbol{k}^{*}\right)=\frac{\int S\left(\mathbf{r}^{*}, \boldsymbol{k}^{*}\right)\left|\boldsymbol{\Psi}_{-\mathbf{k}^{*}}^{\mathbf{S}(+)}\left(\mathbf{r}^{*}, \boldsymbol{k}^{*}\right)\right|^{2}}{\int S\left(\mathbf{r}^{*}, \boldsymbol{k}^{*}\right)} .
$$

$\boldsymbol{k}^{*}$ is the momentum of the first particle in Pair Rest Frame, $\mathbf{r}^{*}=\mathbf{x}_{\mathbf{1}}-\mathbf{x}_{\mathbf{2}}$ is a relative space-time distance of the two particles, $S$ is the source emission function. Usually a spherically symmetric source with size $r_{0}$ is assumed:

$$
S\left(\boldsymbol{r}^{*}\right) \approx \exp \left(-\frac{r^{* 2}}{4 r_{0}^{2}}\right)
$$

In case of $\mathrm{p} \bar{\Lambda}$ system, strong FSI is the only source of the correlation. Thus, the Bethe-Salpeter amplitude takes a form:

$$
\Psi_{-k^{*}}^{S(+)}\left(\mathbf{r}^{*}, \boldsymbol{k}^{*}\right)=e^{-i \boldsymbol{k}^{*} \cdot \boldsymbol{r}^{*}}+f^{S}\left(k^{*}\right) \frac{e^{i k^{*} r^{*}}}{r^{*}}
$$

where $f^{S}$ is the S-wave strong interaction amplitude which may be expressed as:

$$
f^{S}\left(k^{*}\right)=\left(\frac{1}{f_{0}}+\frac{1}{2} d_{0} k^{* 2}-i k^{*}\right)^{-1} .
$$

Complex numbers: $f_{0}$ and $d_{0}$ are the scattering length and the effective radius of the strong interaction, respectively.

Finally, the correlation function which depends only on the strong FSI can be written as [16]:

$$
C\left(\boldsymbol{k}^{*}\right)=1+\sum_{S} \rho_{S}\left[\frac{1}{2}\left|\frac{f^{S}\left(k^{*}\right)}{r_{0}}\right|^{2}\left(1-\frac{d_{0}^{S}}{2 \sqrt{\pi} r_{0}}\right)+\frac{2 \mathfrak{R} f^{S}\left(\boldsymbol{k}^{*}\right)}{\sqrt{\pi} r_{0}} F_{1}\left(2 k^{*} r_{0}\right)-\frac{\mathfrak{J} f^{S}\left(\boldsymbol{k}^{*}\right)}{r_{0}} F_{2}\left(2 k^{*} r_{0}\right)\right]
$$

$F_{1}(z)=\int_{0}^{z} d x e^{x^{2}-z^{2}} / z$ and $F_{2}=\left(1-e^{-z^{2}}\right) / z, \rho_{S}$ is the fraction of pairs in total spin state $\mathrm{S}$. In this work dependence of the correlation on $S$ is neglected and $d_{0}$ is set to zero. The above formula may be directly fitted to the experimental data to extract source size $r_{0}$ as well as strong interaction parameter $f_{0}$. In particular, one has access to imaginary part of the $f_{0}$, describing annihilation process.

\section{Residual correlations}

In collider experiments, when heavy ions collide, a number of particles are produced. They propagate radially from the collision vertex situated in the centre of the detector. Due to the fact that spatial resolution is usually comparable with the distance between trajectory of particles from weak decays and collision point, a meaningful number of secondary baryons cannot be separated from those which were directly produced in the collision. Hence, some pairs analysed in baryon femtoscopy can consist particle(s) coming from weak decays. Correlations in such pairs arise due to interaction of parent particles. Since daughters are measured in detectors, one should apply in Eq. (1) $k^{*}$ and $r^{*}$ calculated between parent particles to correctly describe the process. Next, one or both particles decay with modified $k^{*}$ which corresponds to the one measured in experiment and in terms of which correlation function is expressed. Clearly, the effective correlation observed for a daughter pair will be diluted compared to the original one due to randomness of the weak decay. Such phenomenon is called residual correlations. One cannot neglect this effect when the correlation between parents is 
significant, the fraction of daughter pairs is substantial and the momentum of the decay products in the rest frame of the parent particle (decay momentum) is similar to the correlation width. $\mathrm{p} \bar{\Lambda}$ is the system which meets all the above conditions. Estimation of pair fractions in [10] suggests that only $15 \%$ of $\mathrm{p} \bar{\Lambda}$ pairs are composed of primary particles. The most important contamination sources are $\mathrm{p} \overline{\Sigma^{0}}$ (proton is primary, $\bar{\Lambda}$ is a product of $\bar{\Sigma}^{0}$ decay) and $\Lambda \bar{\Lambda}$ (proton in the final state comes from $\Lambda$ decay and $\bar{\Lambda}$ is primary) $-11 \%$ and $10 \%$, respectively. There are seven more non-negligible sources of residual correlations. Annihilation, realised by non-zero imaginary part of the scattering length, typically leads to an anticorrelation width of the order of hundreds $\mathrm{MeV} / c$. It appears to be no less than decay momenta for considered pairs. For instance, in $\Lambda \rightarrow p+\pi^{-}$decay it is $101 \mathrm{MeV} / c$. The strength of correlation for baryon-antibaryon pairs is generally not known (apart from $\mathrm{p} \overline{\mathrm{p}}$ ). It is determined by the value of the scattering length and the effective range. Nevertheless, assuming that they are not very different than the values for $\mathrm{p} \overline{\mathrm{p}}$, all the pairs mentioned in [10] should be taken into account as a possible contribution of residual correlations.

Residual correlation function from any parent pair feeding down to the $\mathrm{p} \bar{\Lambda}$ correlation may be written as:

$$
C^{X \bar{Y} \rightarrow p \bar{\Lambda}}\left(k_{p \bar{\Lambda}}^{*}\right)=\frac{\int C^{X \bar{Y}}\left(k_{X \bar{Y}}^{*}\right) W\left(k_{X \bar{Y}}^{*}, k_{p \bar{\Lambda}}^{*}\right) d k_{X \bar{Y}}^{*}}{\int W\left(k_{X \bar{Y}}^{*}, k_{p \bar{\Lambda}}^{*}\right) d k_{X \bar{Y}}^{*}},
$$

where $W\left(k_{X \bar{Y}}^{*}, k_{p \bar{\Lambda}}^{*}\right)$ is the probability that parent pair with $k_{X Y}^{*}$ decays into pair with $k_{p \bar{\Lambda}}^{*}$. Examples of $\Lambda \bar{\Lambda}$ and $\Sigma^{+} \overline{\Sigma^{0}}$ residual correlations contributing to $\mathrm{p} \bar{\Lambda}$ along with the normal $\mathrm{p} \bar{\Lambda}$ correlation function are shown in Fig. 1. They are calculated using Eq. (5) and assuming the same radius and strong interaction parameters for all these pairs. Matrices of probability $W\left(k_{X \bar{Y}}^{*}, k_{p \bar{\Lambda}}^{*}\right)$ were calculated using simulations in the Therminator model $[17,18]$. The most notable feature seen in this plot is the fact that at high $k^{*}$ the strength and the shape of residual correlations are similar to the parent one. The dilution resulting from the random nature of decays, dependent on whether one or both particles decayed, affects mainly the low $k^{*}$ region.

The final form of the formula which can be used to fit the $\mathrm{p} \bar{\Lambda}$ experimental correlation function taking into account all residual components can be written in the following way:

$$
C\left(k_{p \bar{\Lambda}}^{*}\right)=1+\lambda_{p \Lambda}\left(C^{p \bar{\Lambda}}\left(k_{p \bar{\Lambda}}^{*}\right)-1\right)+\sum_{X \bar{Y}} \lambda_{X Y}\left(C^{X \bar{Y}}\left(k_{p \bar{\Lambda}}^{*}\right)-1\right) .
$$

The $\lambda_{X Y}$ value refers to fraction of $X Y$ pair contributing to $\mathrm{p} \bar{\Lambda}$ correlation function. Since each (of all nine residual components) $C^{X Y}$ depends on source size, scattering length and effective range, there are too many parameters to obtain reliable results. Several assumptions must be introduced. Firstly, the effective range is fixed to 0 [10]. Furthermore, source sizes of all considered systems are equalised. This is justified following hydrodynamics prediction saying that radius is approximately proportional to $1 / \sqrt{\left\langle m_{\mathrm{T}}\right\rangle}$. Baryon pairs studied here have relatively large and comparable $\left\langle m_{\mathrm{T}}\right\rangle$. Thus, radii should not differ much. Another assumptions can be made regarding the values of the scattering length. As a default case, the scattering length is the free fit parameter, equal for all baryon-antibaryon pairs, excluding $\mathrm{p} \overline{\mathrm{p}}$ for which the value is fixed to the measured one [19].

\section{Reanalysis of STAR data}

The STAR collaboration published $\mathrm{p} \bar{\Lambda}$ femtoscopic correlation functions corrected for purity, i.e. scaled by the fraction of primary $\mathrm{p} \bar{\Lambda}$ pairs $(15 \%)$. However, such procedure may be applied only if there is no correlation for other pairs. It was shown that this is not the case due to residual correlations 


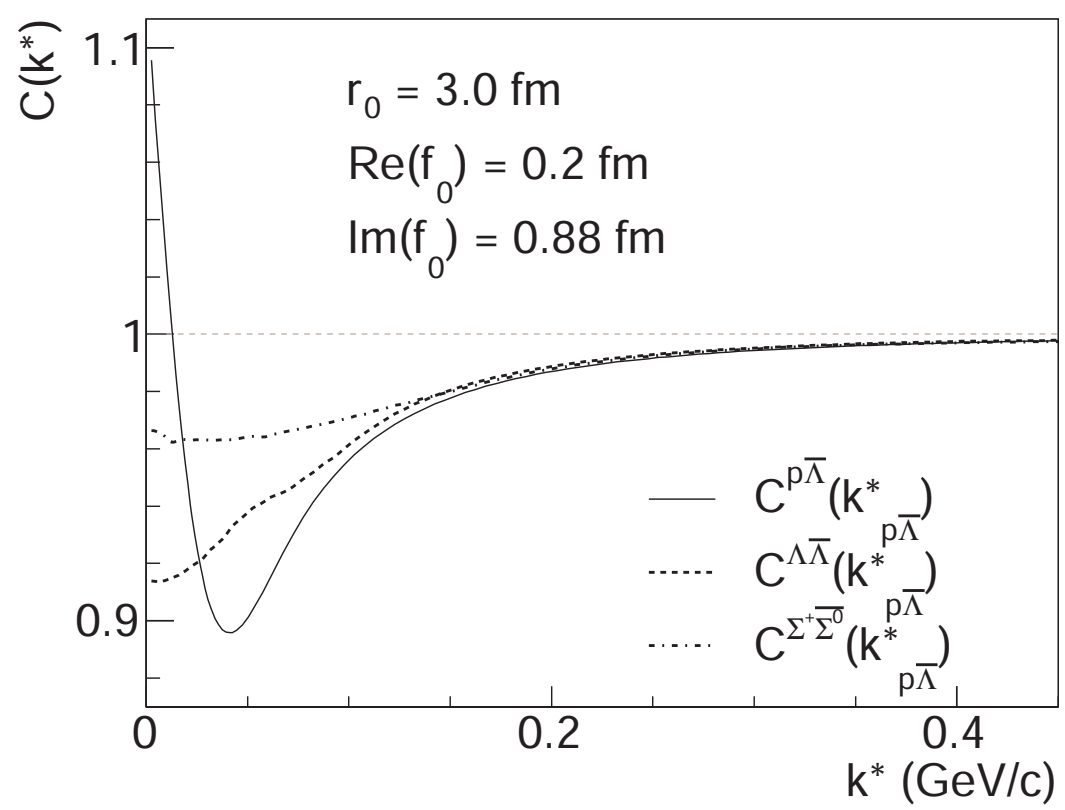

Figure 1. Theoretical correlation function for $\mathrm{p} \bar{\Lambda}$ with two examples of residual correlation functions for $\Lambda \bar{\Lambda}$ and $\Sigma^{+} \overline{\Sigma^{0}}$ pairs. Source size and strong interaction parameters are set arbitrarily.

which cannot be neglected for the $\mathrm{p} \bar{\Lambda}$ system. Hence, for this analysis, we have undone the correction procedure so that the Eq. (7) could be used for the fitting procedure. $\chi^{2}$ minimisation is used.

In Fig. 2 the STAR p $\bar{\Lambda}$ correlation function with the fit taking into account residual correlation is presented. In this scenario, values of scattering length are assumed to be equal among all baryonantibaryon pairs except for $\mathrm{p} \overline{\mathrm{p}}$. The fitted source size is $r_{0}=(2.83 \pm 0.12) \mathrm{fm}$ and is noticeably larger than the value from [10]. Moreover, it is consistent with the result for $\mathrm{p} \Lambda$ system obtained there. Extracted scattering length is $f_{0}=[(0.49 \pm 0.21)+i(1.00 \pm 0.21)] \mathrm{fm}$. This is compatible with known value for $\mathrm{p} \overline{\mathrm{p}}$. Therefore, the assumption of similar annihilation (realised by the non-zero imaginary part of $f_{0}$ ) among all baryon-antibaryon pairs is supported by STAR femtoscopic data. Including the contribution of residual correlations turns out to be crucial in studying $\mathrm{p} \bar{\Lambda}$ femtoscopic correlations.

To check the proposed formalism for dealing with residual correlations in $\mathrm{p} \bar{\Lambda}$ femtoscopic correlation, several tests were performed. Firstly, the STAR method was repeated, i.e. no residual correlations were considered in the fit. STAR results are reproduced as presented in Fig. 3. Comparing these results with the default case (with all residual correlations included), one can notice that real part of the scattering length changes the sign whereas imaginary part remains similar in magnitude.

Another validation method is based on the assumption that values of the scattering length parameters are scaled with $\sqrt{s}$ of the pair instead of $k^{*}$, following [20]. It means there are still only two free fit parameters corresponding to the $f_{0}$ (real and imaginary part), but values change from pair to pair depending on the factor decreasing with the pair mass. Interestingly, the results of the fit indicate the value of the radius similar to the default case, whereas the imaginary part of the scattering length is 


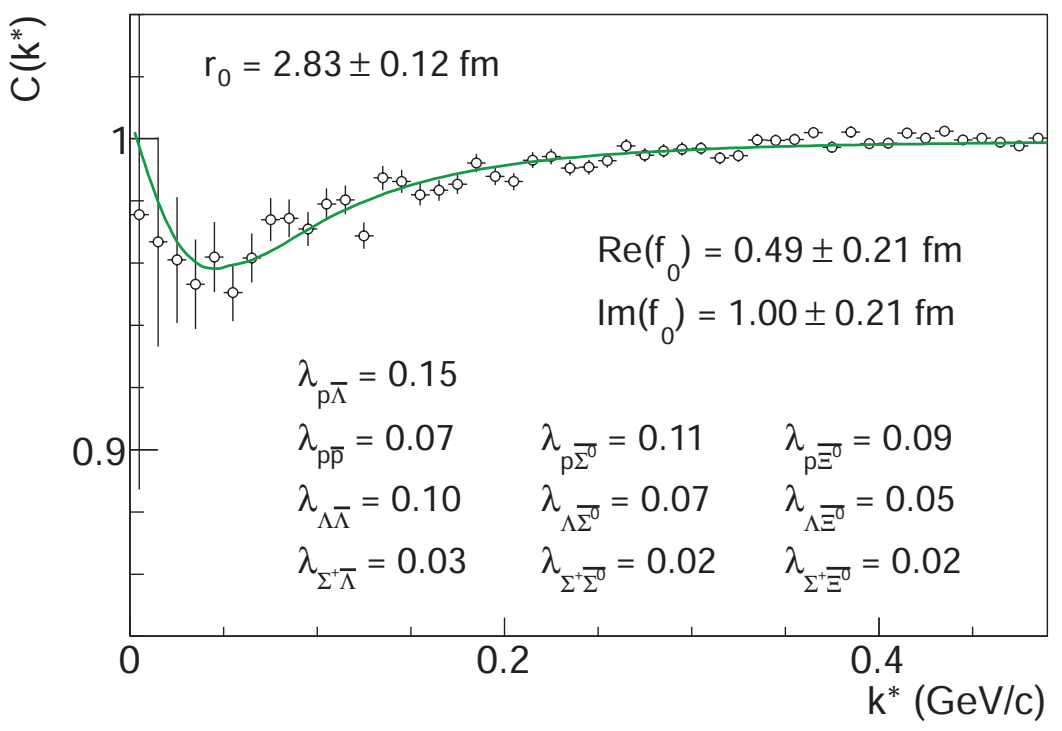

Figure 2. $\mathrm{p} \bar{\Lambda}$ correlation function measured in STAR fitted with Eq. (7) (taking into account all residual components).

substantially bigger. What is more, the extracted value of $\mathfrak{J} f_{0}$ for $\mathrm{p} \bar{\Lambda}$ is bigger that the value for $\mathrm{p} \overline{\mathrm{p}}$. Such method is hence disfavoured since it leads to the non-monotonic behaviour of the cross-section with the mass of the pair.

Finally, we consider the case that the annihilation occurs only for particle-antiparticle pairs, i.e. particles where the quark content is opposite. In practice, it means that only residual correlations from $\mathrm{p} \overline{\mathrm{p}}, \Lambda \bar{\Lambda}$ and $\Lambda \bar{\Sigma}_{0}$ are included in the fitting formula from Eq. (7). As a result of such test, one obtains relatively small radius $r_{0}=1.5 \pm 0.1 \mathrm{fm}$, comparable with the case without residual correlations. As it seems to be nonphysically small value, the conclusion can be made that this study supports the concept that annihilation (non-zero imaginary part of the scattering length) occurs for all baryon-antibaryon pairs.

\section{Summary}

In this talk the method for dealing with residual correlations in the femtoscopic analysis of baryonantibaryon pairs is presented. This formalism is tested on $\mathrm{p} \bar{\Lambda}$ correlation function measured by the STAR experiment in Au-Au collisions at centre-of-mass energy per nucleon pair $\sqrt{s_{\mathrm{NN}}}=200 \mathrm{GeV}$. It is shown that taking into account residual correlations is vital for $\mathrm{p} \bar{\Lambda}$ femtoscopy. In particular, with such an approach source size extracted from $\mathrm{p} \bar{\Lambda}$ analysis is comparable with the one from $\mathrm{p} \Lambda$ studies as well as lighter pair types and model calculations. Hence, it resolves the riddle of the unexpectedly small $\mathrm{p} \bar{\Lambda}$ radius. Furthermore, the value of the scattering length parameters for baryon-antibaryon 


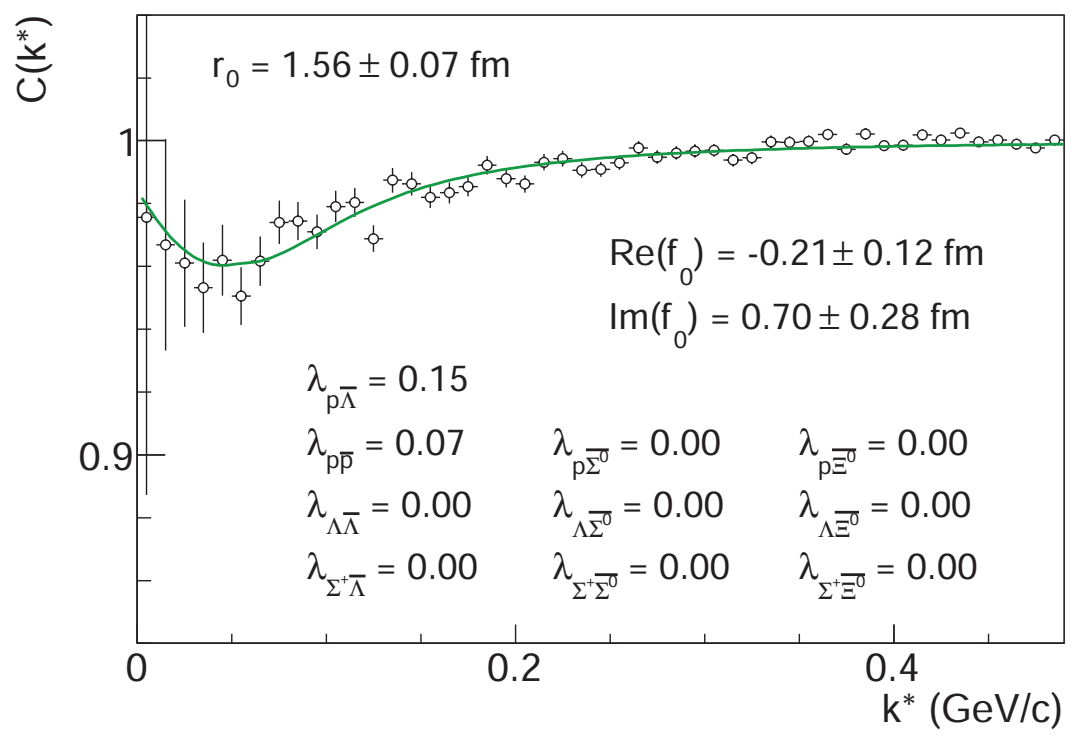

Figure 3. $p \bar{\Lambda}$ correlation function measured in STAR fitted with Eq. (7) (without any residual component).

pairs are estimated. The data favours the case in which all baryon-antibaryon pairs analysed here have comparable cross-section for annihilation. The formalism presented in this work may be effectively applied to measure currently unknown parameters of strong interactions for pairs including e.g. $\Lambda$ and $\Sigma$ baryons once more precise data on baryon femtoscopic correlation functions will be available.

\section{References}

[1] A. Kisiel, H. Zbroszczyk, M. Szymanski, Phys.Rev. C89, 054916 (2014), 1403.0433

[2] G. Kopylov, M. Podgoretsky, Sov.J.Nucl.Phys. 15, 219 (1972)

[3] G. Kopylov, V. Lyuboshits, M. Podgoretsky (1974)

[4] M.A. Lisa, S. Pratt, R. Soltz, U. Wiedemann, Ann.Rev.Nucl.Part.Sci. 55, 357 (2005), nucl-ex/0505014

[5] K. Aamodt et al. (ALICE), Phys.Lett. B696, 328 (2011), 1012 . 4035

[6] H. Gos, The European Physical Journal C 49, 75 (2007)

[7] L. Adamczyk et al. (STAR), Phys.Rev. C88, 034906 (2013), 1302 . 3168

[8] B. Abelev et al. (STAR), Phys.Rev. C74, 054902 (2006), nucl-ex/0608012

[9] M.P. Szymanski (ALICE Collaboration), Nucl.Phys. A904-905, 447c (2013), 1211. 3288

[10] J. Adams et al. (STAR Collaboration), Phys.Rev. C74, 064906 (2006), nucl-ex/0511003

[11] A. Makhlin, Y. Sinyukov, Z.Phys. C39, 69 (1988)

[12] A. Kisiel, M. Galazyn, P. Bozek (2014), 1409. 4571 
[13] R. Lednicky, Phys.Part.Nucl. 40, 307 (2009), nucl-th/0501065

[14] K. Olive et al. (Particle Data Group), Chin.Phys. C38, 090001 (2014)

[15] B. Abelev et al. (ALICE Collaboration), Phys.Rev. C88, 044910 (2013), 1303.0737

[16] R. Lednicky, V. Lyuboshits, Sov.J.Nucl.Phys. 35, 770 (1982)

[17] A. Kisiel, T. Taluc, W. Broniowski, W. Florkowski, Comput.Phys.Commun. 174, 669 (2006), nucl-th/0504047

[18] M. Chojnacki, A. Kisiel, W. Florkowski, W. Broniowski, Comput.Phys.Commun. 183, 746 (2012), 1102.0273

[19] C. Batty, Rept.Prog.Phys. 52, 1165 (1989)

[20] M. Bleicher, E. Zabrodin, C. Spieles, S. Bass, C. Ernst et al., J.Phys. G25, 1859 (1999), hep-ph/9909407 